\title{
Influence of hot plasma pressure on the global structure of Saturn's magnetodisk
}

\author{
N. Achilleos, ${ }^{1,2}$ P. Guio, ${ }^{1,2}$ C. S. Arridge, ${ }^{2,3}$ N. Sergis, ${ }^{4}$ R. J. Wilson, ${ }^{5,6}$ M. F. Thomsen, ${ }^{5}$ \\ and A. J. Coates ${ }^{2,3}$
}

Received 18 August 2010; revised 9 September 2010; accepted 15 September 2010; published 19 October 2010.

[1] Using a model of force balance in Saturn's disk-like magnetosphere, we show that variations in hot plasma pressure can change the magnetic field configuration. This effect changes (i) the location of the magnetopause, even at fixed solar wind dynamic pressure, and (ii) the magnetic mapping between ionosphere and disk. The model uses equatorial observations as a boundary condition-we test its predictions over a wide latitude range by comparison with a Cassini highinclination orbit of magnetic field and hot plasma pressure data. We find reasonable agreement over time scales larger than the period of Saturn kilometric radiation (also known as the camshaft period). Citation: Achilleos, N., P. Guio, C. S. Arridge, N. Sergis, R. J. Wilson, M. F. Thomsen, and A. J. Coates (2010), Influence of hot plasma pressure on the global structure of Saturn's magnetodisk, Geophys. Res. Lett., 37, L20201, doi:10.1029/2010GL045159.

\section{Introduction}

[2] Saturn's equatorial, rotating plasma disk is threaded by a magnetic field which changes from a quasi-dipolar geometry, at distances $\lesssim 12 R_{S}$ (Saturn's radius $R_{S}=60280 \mathrm{~km}$ ), to a "magnetodisk" in the outer region, where the radial field component is dominant at distances $\sim 1-2 R_{S}$ from the equator [e.g., Arridge et al., 2007]. This magnetodisk is supported by an azimuthal ring current whose solenoid-like field adds to the planetary dipole. In early studies, the ring current was represented by a bound annular region with four free parameters: a uniform half-thickness, inner and outer radii, and a current scaling parameter [e.g., Connerney et al., 1981]. In this model, current density $J_{\phi}$ was assumed inversely proportional to cylindrical radial distance $\rho$. This formalism ("CAN81 disk") was recently adapted to analyze Cassini magnetic data, establishing how ring current parameters depend on magnetopause size [Bunce et al., 2007].

[3] More recently, a formalism for calculating selfconsistent field and plasma distributions for the Jovian magnetodisk [Caudal, 1986] has been implemented for Saturn [Achilleos et al., 2010, hereafter A10]. This approach

\footnotetext{
${ }^{1}$ Department of Physics and Astronomy, University College London, London, UK.

${ }^{2}$ Centre for Planetary Sciences at UCL/Birkbeck, London, UK

${ }^{3}$ Mullard Space Science Laboratory, Dorking, UK.

${ }^{4}$ Office for Space Research and Technology, Academy of Athens, Athens, Greece.

${ }^{5}$ Space Science and Applications, Los Alamos National Laboratory, Los Alamos, New Mexico, USA.

${ }^{6}$ Laboratory of Atmospheric and Space Physics, University of Colorado at Boulder, Boulder, Colorado, USA.
}

Copyright 2010 by the American Geophysical Union. 0094-8276/10/2010GL045159 integrates equations of force balance throughout a rotating, axisymmetric magnetosphere (section 2). In section 3, we use A10's model to show that the variability of hot plasma pressure, as observed, affects the magnetospheric field structure-increased hot plasma content generally producing a more "inflated" or "disk-like" field geometry. To test the model's high-latitude structure, we compare its outputs with field and particle data from the Rev 40 high-inclination orbit of Cassini. We discuss the agreement between model and data and summarize our conclusions in section 4 .

\section{Magnetodisk Model Inputs}

[4] We have improved A10's representation of the cold equatorial ion temperature over $10-25 R_{S}$, by combining temperatures for protons and water group ions from McAndrews et al. [2009] ( 10-30 $\left.\mathrm{R}_{\mathrm{S}}\right)$ and Wilson et al. [2008] (5-10 $R_{S}$ ), who analyzed energy spectra acquired by the ion mass spectrometer (IMS), a subsystem of the Cassini Plasma Spectrometer (CAPS) [Young et al., 2004]. Temperature moments versus equatorial distance, along with quartic fits, are shown in Figures 1a and $1 \mathrm{~b}$ (fit coefficients available in the auxiliary material). ${ }^{1}$ A10's initial study used only Wilson et al.'s [2008] temperatures, with parabolic extrapolation beyond $10 \mathrm{R}_{\mathrm{S}}$. Using the updated profiles, we derived the single plasma temperature $T_{c}(\rho)$ required by the model, whose field lines are loci of constant, isotropic temperature. This was done as by A10, by taking weighted average moments for each ion species, then combining these according to a prescribed plasma composition.

[5] To model hot plasma content, we used the equatorial product $P_{h}(\rho) V_{\alpha}(\rho)$ of hot plasma pressure (assumed constant along a field line) and unit flux tube volume [Caudal, 1986; A10]. We extended the Cassini Magnetospheric Imaging Instrument (MIMI) pressure dataset used in A10's initial study [Sergis et al., 2007] to cover 23 near-equatorial orbits in total, between October, 2004 and March, 2006. The hot pressure arises from ions (mainly $\mathrm{H}^{+}$and $\mathrm{O}+$ ) with energies $>3 \mathrm{keV}$ [e.g., Sergis et al., 2009]. The moments are computed in the spacecraft frame, from an incomplete sampling of the phase space. However, for $\rho>12 \mathrm{R}_{\mathrm{S}}$, energetic ions have typical thermal speeds $\sim 10$ times higher than the plasma flow speed. The resulting hot pressures are uncertain by factors of $\sim 2-3$, within the scatter of the presented data. We used pressure moments sampled every 10 minutes, taking: (i) absolute latitudes $<5^{\circ}$, (ii) Saturn local times 09:00-15:00, and (iii) locations between each orbit's last inbound and next outbound magnetopause crossings.

\footnotetext{
${ }^{1}$ Auxiliary materials are available in the HTML. doi:10.1029/ 2010GL045159.
} 


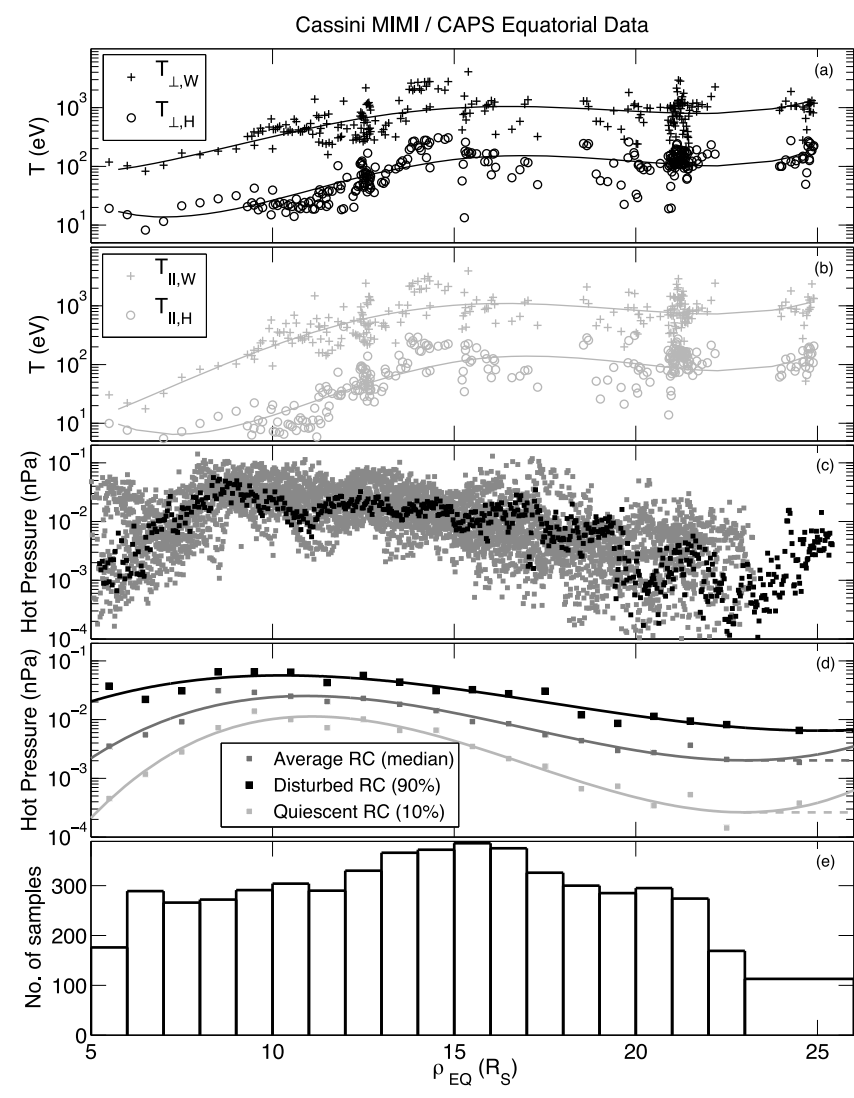

Figure 1. (a, b) Perpendicular and parallel temperature moments (with respect to assumed-southward magnetic field) for protons $(\mathrm{H}$, circles) and water group ions (W,crosses), from Wilson et al. [2008] and McAndrews et al. [2009]. Solid curves show quartic fits. (c) Hot plasma pressure derived from the Cassini Magnetospheric Imaging Instrument (MIMI) data. Gray squares cover 23 Cassini orbits, black squares are from Rev 3. (d) Median and quantile pressures computed for bins in Figure 1e. Solid curves are cubic fits used to calculate $P_{h} V_{\alpha}$ (see text). (e) Radial distance bins and number of samples used.

[6] All hot pressures are shown as gray symbols in Figure 1c, collapsed onto a single profile in $\rho$. For comparison, data for Cassini Rev 3 (February 14-23, 2005) are overlain in black (data from this orbit were previously analyzed by A10). Figure $1 \mathrm{~d}$ shows the median, $10 \%$ and $90 \%$ quantiles. Solid curves are cubic fits to these parameters, which correspond to "average", "disturbed" and "quiescent" ring current states (following Sergis et al. [2007] and A10). We assumed a zero-slope profile past $\sim 23 R_{S}$ (dashed lines in Figure 1d), however this did not affect our conclusions. Repeating this parametrization, incorporating all local times, elevated the quantile pressures by $\sim 40 \%$.

\section{Comparison of Magnetodisk Models and Observations}

[7] Figure 2 illustrates the model magnetodisk's response to ring current state. Left and right columns show calculations for fixed magnetopause radius $\mathrm{R}_{\mathrm{MP}}=25 R_{S}$ (average value for Saturn [Achilleos et al., 2008]), but for quiescent and disturbed states (Figure 1d). Figure 2a shows that field lines in the disturbed model become more radially "stretched" for $\rho$ $\sim 10-15 \mathrm{R}_{\mathrm{S}}$. The labeled field lines intersect northern ionospheric colatitudes $\theta_{i}=15^{\circ}, 17^{\circ}$ and $19^{\circ}$. These values are first order estimates, since we assume a centered dipole for the planet's internal field; Nichols et al. [2009] have used auroral observations and a more realistic internal field model to show that the northern auroral oval has a radius $\sim 1.5^{\circ}$ smaller than the southern. The model magnetopause shielding field is valid for latitudes $\lesssim 50^{\circ}$ [ Caudal, 1986], and the labeled field lines exceed this limit for radial distances $\lesssim 7 R_{S}$. Increased hot plasma content expands the equatorial distance of the labeled field lines by $\sim 3-4 \mathrm{R}_{\mathrm{S}}$. The ionospheric colatitudes $15-17^{\circ}$ are $\lesssim 1^{\circ}$ from the observed equatorward boundary of the northern auroral oval [Nichols et al., 2009]. Thus internal re-configuration of hot plasma, at fixed $\mathrm{R}_{\mathrm{MP}}$, can influence ionosphere-disk magnetic mapping and equatorial width of the auroral current layer. A similar effect was noted by Grodent et al. [2008] from auroral observations of Jupiter.

[8] In Figure 2b, we plot equatorial magnetic and plasma pressure for both models. Field strength at $\rho \gtrsim 15 R_{S}$ for the disturbed disk is more uniform than the quiescent model. Total model plasma pressure (disturbed) agrees well, as shown, with that of Sergis et al. [2010, hereafter S10]; derived directly from Cassini Plasma Spectrometer (CAPS) and Cassini Magnetospheric Imaging Instrument (MIMI) pressures). The radial gradient of our profile differs from that of S10, however, which has consequences for azimuthal current (see below). Our disturbed disk's maximum plasma $\beta$ of $\sim 6.5$ at $\rho \sim 14 \mathrm{R}_{\mathrm{S}}$ is consistent with S10's $\beta_{\max } \sim 3-10$ for $\rho \sim 11-14 \mathrm{R}_{\mathrm{S}}$.

[9] Figure 2c compares equatorial volume forces. Again, the smaller gradient in the disturbed disk's magnetic pressure for $\rho \gtrsim 15 \mathrm{R}_{\mathrm{S}}$ is evident. The corresponding increase in total plasma pressure gradient is mainly balanced by increased curvature force of the more disk-like field. In the outer model disk, plasma pressure gradient becomes smaller than centrifugal force within a few $R_{S}$ of the magnetopause. This result, arising from our improved pressure representation, differs greatly from A10's "transition distance" of $\sim 12 \mathrm{R}_{\mathrm{S}}$, but agrees with Arridge et al. [2007], who deduced plasma pressure from Cassini magnetic data during current sheet encounters.

[10] Figure $2 \mathrm{~d}$ compares the modeled equatorial azimuthal current density, associated with pressure gradient and centrifugal force, with S10's data-derived values. Considering centrifugal (inertial) current, the model is in reasonable agreement with the data for $\rho \gtrsim 6 \mathrm{R}_{\mathrm{S}}$. The discrepancy is mainly due to the difference between our approach (see A10) and S10 for computing currents. For centrifugal current, we assume A10's polynomial fit to plasma angular velocity $\omega$ obtained from high-energy particle anisotropies [Kane et al., 2008], combined with inner magnetospheric values from Wilson et al. [2008]. Combining our magnetic field model and profile for ion flux tube content produces the centrifugal term. S10 employed Cassini Plasma Spectrometer (CAPS) density and velocity moments from Wilson et al. [2008] and McAndrews et al. [2009], and their own Cassini Plasma Spectrometer (CAPS) ion moment computations, in order to derive and fit centrifugal volume force as a function of $\rho$. The ratio of our centrifugal force to that of S10 ranges from $\sim 2$ at $\sim 6 R_{S}$ to $\sim 0.5$ at $\sim 12 R_{S}$.

[11] Considering pressure gradient, we recall (discussion of Figure 2b) considerable differences in plasma pressure 


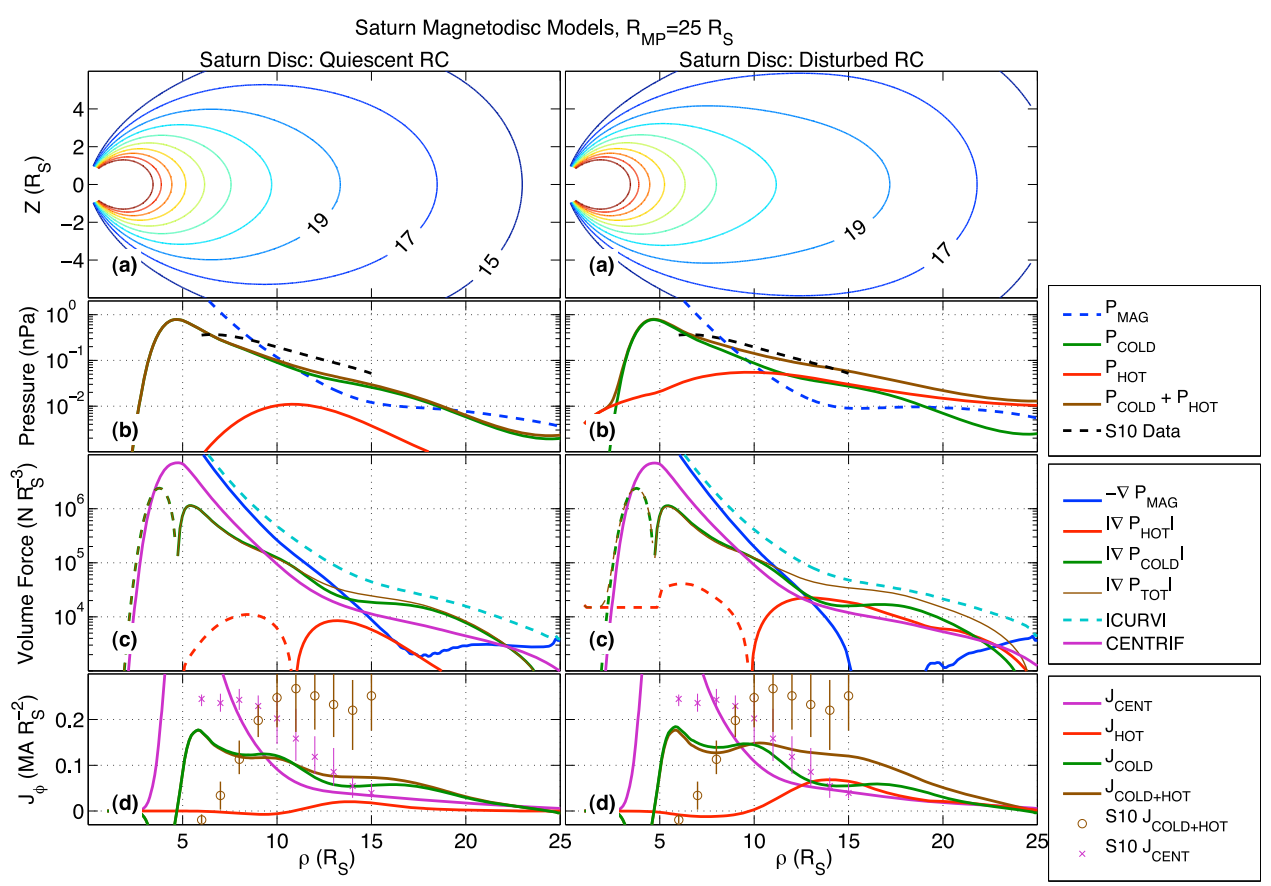

Figure 2. Model outputs for magnetopause radius $\mathrm{R}_{\mathrm{MP}}=25 \mathrm{R}_{\mathrm{S}}$, and for (left) quiescent and (right) disturbed ring current state. (a) Magnetic field lines, partially labeled with the colatitude $\theta_{i}$ (in degrees) of their northern ionospheric footpoint $\left(2^{\circ}\right.$ steps in $\left.\theta_{i}\right)$. (b) Equatorial pressure. Color code indicates magnetic or plasma pressure (cold, hot and total), dashed black curve is from S10. (c) Equatorial volume forces, colored according to physical origin. Line style indicates radially outward (solid) or inward (dashed) force. (d) Equatorial azimuthal current density, colored according to physical origin, compared with the medians (symbols), 10\% and 90\% quantiles (vertical bars) of the data-derived currents of S10 (1 $R_{S}$ window).

gradients between our model and S10's values. Thus for $\rho \gtrsim 8 \mathrm{R}_{\mathrm{S}}$, our disturbed model's total pressure current seems reasonably consistent with S10's lower limits. Current density exceeds inertial at $\sim 8 \mathrm{R}_{\mathrm{S}}$ in both model and data. For $\rho \lesssim$ $8 \mathrm{R}_{\mathrm{S}}$, Sergis et al. [2010] find $>25 \%$ of total current arises from pressure anisotropy. This is not included in our model at present.

[12] In Figures $3 \mathrm{a}$ and 3b, we compare model outputs with hot plasma and magnetic pressures for Cassini's Rev 40 orbit from the Cassini Magnetospheric Imaging Instrument (MIMI) and Cassini magnetometer (MAG) [Dougherty et al., 2004]. The model assumes constant hot pressure $P_{h}$ along field lines, hence the change in local pressure with altitude $Z$ (from the equator) is equivalent to the change in the value $P_{h 0}$ at the equatorial crossings of the field lines sampled by the spacecraft. Values of $\mathrm{R}_{\mathrm{MP}}$ were determined by $\mathrm{A} 10$, who modeled individual field components during this orbit.

[13] Hot pressures near the nightside current sheet crossing (CSX1) are well captured by a quiescent disk model, while the dayside crossing (CSX2) requires an "intermediate" state. Sharp "dropouts" in pressure near days $\sim 67.5$ and $\sim 70$ (decimal day of year) are also seen in the model, and coincide with the spacecraft's exit from and entry into the polar cap, characterized by open magnetic flux and relative absence of plasma. This region manifests in the model as field lines which do not cross the equator inside the magnetopause, hence are assumed to be devoid of plasma. Polar cap boundary crossings are also seen in nightside models, near days $\sim 63.9$ and $\sim 65.9$. For these local times, however, observed pressures are small, near noise level, and could obscure any dropout signature. Quasi-periodic "pulsations" are present in plasma and magnetic data, which we emphasize with vertical lines spaced by the nominal $10.75 \mathrm{hr}$ planetary rotation period. These features are due to the quasi-periodic "camshaft" oscillations in the Kronian magnetosphere, whose period agrees with that of the Saturn kilometric radiation [e.g., Southwood and Kivelson, 2007; Kurth et al., 2007; Provan et al., 2009].

[14] The change in magnetic pressure when crossing the current sheet is greater at CSX1 than $\mathrm{CSX} 2$. CSX1 occurs at $\rho$ $\sim 15 R_{S}$ in the "middle magnetosphere" where the field lines are most disk-like, hence produce a strong contrast in field strength between the inside and outside of the sheet (Figure 2a). CSX2 occurs at $\rho \sim 20 R_{S}$ towards the "outer region" where field lines are more "blunt" (larger radius of curvature), this contrast is thus reduced, and the cold plasma sheet is thicker (A10). Models with more disturbed disk and/or larger $R_{M P}$ show a change in magnetic pressure comparable to that seen at CSX1. However, the observed sheet transit is more rapid than the model analog, perhaps due to plasma sheet motion linked to camshaft oscillations.

[15] Figure 3c is a Cassini Plasma Spectrometer (CAPS) electron spectrogram for the same orbit. The decrease in cold electron plasma (counts) with distance from the equator is more pronounced near CSX1 than CSX2. This aspect reflects global force balance, which allows the cold population to maintain higher pressure for a given $Z$ in regions where the field lines have larger curvature radii, and centrifugal confinement is weaker [e.g., A10; Caudal, 1986]. Nightside data are better matched by a more quiescent disk and larger $\mathrm{R}_{\mathrm{MP}}$, both possible signatures of a thinner nightside plasma sheet, as described by Sergis et al. [2009]. The magnetosheath population seen after the outbound magnetopause crossing (MPX, early on day 70) shows peak energies similar to those 


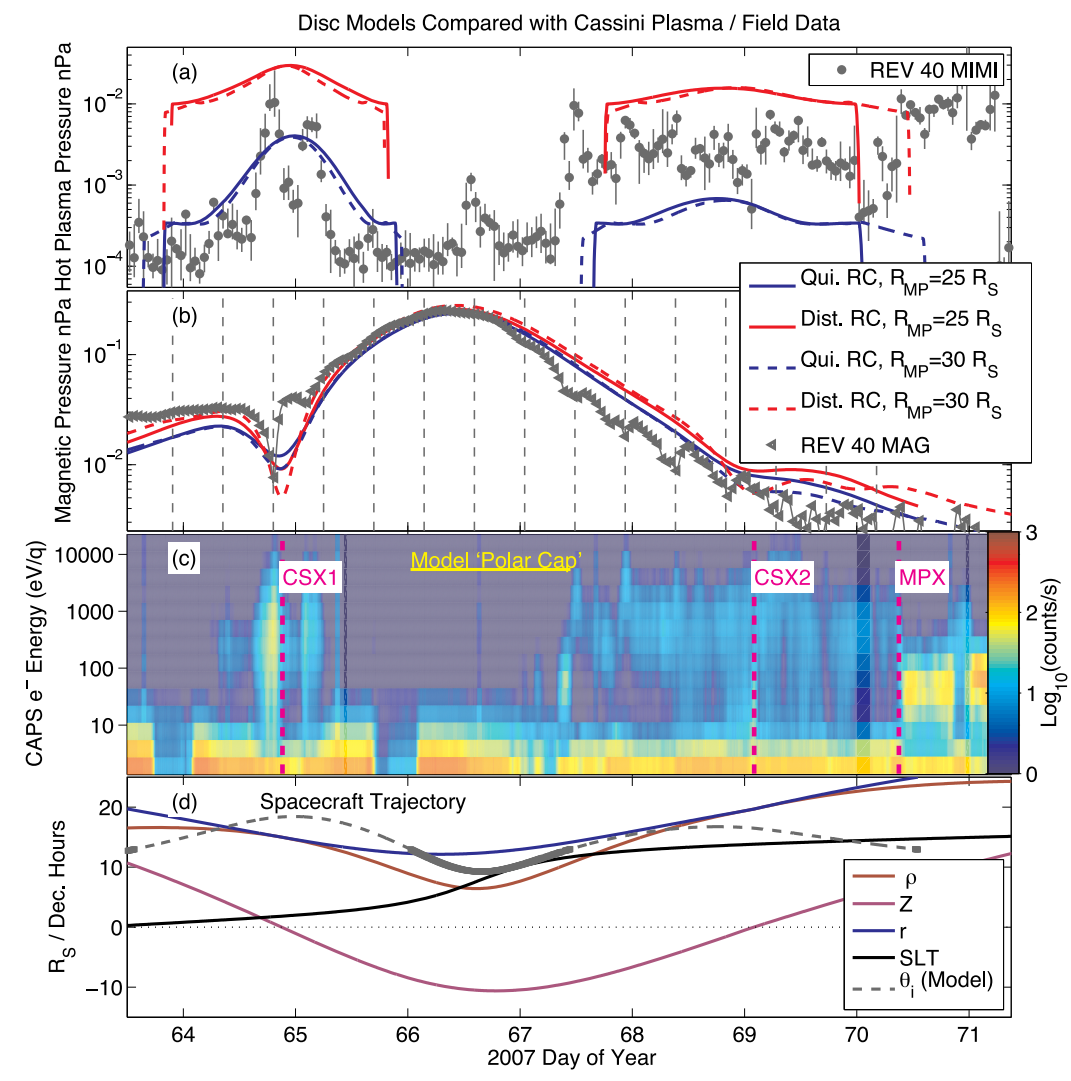

Figure 3. (a) Hot plasma pressure versus time for Cassini's Rev 40 orbit (symbols are medians, vertical bars show $25 \%$ and $75 \%$ quantiles, for a one-hour window). Disk model pressures are overlaid (see code in Figure $3 b$ ). (b) Observed and modeled magnetic pressure for Rev 40, with Cassini magnetometer (MAG) data shown as symbols and model curves color- and linecoded according to magnetopause radius and ring current activity. Thin vertical dashed lines are spaced by the planetary rotation period. (c) Cassini Plasma Spectrometer (CAPS) electron spectrogram (one-hour average from Anode 5), with current sheet (CSX) and magnetopause (MPX) crossings marked. Horizontal line shows the model polar cap interval. (d) Spacecraft trajectory, showing cylindrical and spherical radial distance $\rho$ and $r$, vertical coordinate $Z$, local time SLT and model colatitude $\theta_{i}$ of northern magnetic footpoint.

near the exit from the polar cap (via the dayside cusp) at day 67.5. Trajectory information in Figure $3 \mathrm{~d}$ includes northern ionospheric colatitude $\theta_{i}$ magnetically conjugate to Cassini's location. Regions with $\theta_{i}<13^{\circ}$ are shown as a solid curve (also shown in Figure 3c), and represent a model polar cap, located on field lines poleward of the auroral oval boundary [Badman et al., 2006].

\section{Conclusions}

[16] Saturn's magnetospheric field can be significantly modified by internal changes in hot plasma pressure. Magnetic mapping between ionosphere and disk is also dependent on internal plasma state (as well as external solar wind pressure [e.g., Bunce et al., 2008]). This result suggests a corresponding influence on the distribution of currents which flow between these regions and their associated auroral emissions. Comparing our models with both field and plasma data allows identification of ring current state and its magnetic signatures.

[17] Acknowledgments. We acknowledge the continued collaboration of the Cassini magnetometer (MAG) and plasma (CAPS, MIMI) instrument teams. CSA was supported by an STFC Postdoctoral Fellowship under grant ST/G007462/1.

\section{References}

Achilleos, N., C. S. Arridge, C. Bertucci, C. M. Jackman, M. K. Dougherty, K. K. Khurana, and C. T. Russell (2008), Large-scale dynamics of Saturn's magnetopause: Observations by Cassini, J. Geophys. Res., 113, A11209, doi:10.1029/2008JA013265.

Achilleos, N., P. Guio, and C. S. Arridge (2010), A model of force balance in Saturn's magnetodisc, Mon. Not. R. Astron. Soc., 401, 2349-2371, doi:10.1111/j.1365-2966.2009.15865.x.

Arridge, C. S., C. T. Russell, K. K. Khurana, N. Achilleos, N. André, A. M. Rymer, M. K. Dougherty, and A. J. Coates (2007), Mass of Saturn's magnetodisc: Cassini observations, Geophys. Res. Lett., 34, L09108, doi:10.1029/2006GL028921.

Badman, S. V., S. W. H. Cowley, J. Gérard, and D. Grodent (2006), A statistical analysis of the location and width of Saturn's southern auroras, Ann. Geophys., 24, 3533-3545.

Bunce, E. J., S. W. H. Cowley, I. I. Alexeev, C. S. Arridge, M. K. Dougherty, J. D. Nichols, and C. T. Russell (2007), Cassini observations of the variation of Saturn's ring current parameters with system size, J. Geophys. Res., 112, A10202, doi:10.1029/2007JA012275.

Bunce, E. J., C. S. Arridge, S. W. H. Cowley, and M. K. Dougherty (2008), Magnetic field structure of Saturn's dayside magnetosphere and its mapping to the ionosphere: Results from ring current modeling, J. Geophys. Res., 113, A02207, doi:10.1029/2007JA012538.

Caudal, G. (1986), A self-consistent model of Jupiter's magnetodisc including the effects of centrifugal force and pressure, J. Geophys. Res., 91, 4201-4221, doi:10.1029/JA091iA04p04201.

Connerney, J. E. P., M. H. Acuna, and N. F. Ness (1981), Saturn's ring current and inner magnetosphere, Nature, 292, 724-726, doi:10.1038/ $292724 a 0$.

Dougherty, M. K., et al. (2004), The Cassini magnetic field investigation, Space Sci. Rev., 114, 331-383, doi:10.1007/s11214-004-1432-2. 
Grodent, D., J.-C. Gérard, A. Radioti, B. Bonfond, and A. Saglam (2008), Jupiter's changing auroral location, J. Geophys. Res., 113, A01206, doi:10.1029/2007JA012601.

Kane, M., D. G. Mitchell, J. F. Carbary, S. M. Krimigis, and F. J. Crary (2008), Plasma convection in Saturn's outer magnetosphere determined from ions detected by the Cassini INCA experiment, Geophys. Res. Lett. 35, L04102, doi:10.1029/2007GL032342.

Kurth, W. S., A. Lecacheux, T. F. Averkamp, J. B. Groene, and D. A. Gurnett (2007), A Saturnian longitude system based on a variable kilometric radiation period, Geophys. Res. Lett., 34, L02201, doi:10.1029/ 2006 GL028336.

McAndrews, H. J., et al. (2009), Plasma in saturn's nightside magnetosphere and the implications for global circulation, Planet. Space Sci., 57, 1714-1722, doi:10.1016/j.pss.2009.03.003.

Nichols, J. D., et al. (2009), Saturn's equinoctial auroras, Geophys. Res. Lett., 36, L24102, doi:10.1029/2009GL041491.

Provan, G., D. J. Andrews, C. S. Arridge, A. J. Coates, S. W. H. Cowley, S. E. Milan, M. K. Dougherty, and D. M. Wright (2009), Polarization and phase of planetary-period magnetic field oscillations on highlatitude field lines in Saturn's magnetosphere, J. Geophys. Res., 114, A02225, doi:10.1029/2008JA013782.

Sergis, N., S. M. Krimigis, D. G. Mitchell, D. C. Hamilton, N. Krupp, B. M. Mauk, E. C. Roelof, and M. Dougherty (2007), Ring current at Saturn: Energetic particle pressure in Saturn's equatorial magnetosphere measured with Cassini/MIMI, Geophys. Res. Lett., 34, L09102, doi:10.1029/2006GL029223.

Sergis, N., S. M. Krimigis, D. G. Mitchell, D. C. Hamilton, N. Krupp, B. H. Mauk, E. C. Roelof, and M. K. Dougherty (2009), Energetic particle pressure in Saturn's magnetosphere measured with the Magnetospheric Imaging Instrument on Cassini, J. Geophys. Res., 114, A02214, doi:10.1029/2008JA013774.

Sergis, N., et al. (2010), Particle pressure, inertial force, and ring current density profiles in the magnetosphere of Saturn, based on Cassini measurements, Geophys. Res. Lett., 37, L02102, doi:10.1029/ 2009GL041920.

Southwood, D. J., and M. G. Kivelson (2007), Saturnian magnetospheric dynamics: Elucidation of a camshaft model, J. Geophys. Res., 112, A12222, doi:10.1029/2007JA012254.

Wilson, R. J., R. L. Tokar, M. G. Henderson, T. W. Hill, M. F. Thomsen, and D. H. Pontius Jr. (2008), Cassini plasma spectrometer thermal ion measurements in Saturn's inner magnetosphere, J. Geophys. Res., 113, A12218, doi:10.1029/2008JA013486.

Young, D. T., et al. (2004), Cassini Plasma Spectrometer Investigation, Space Sci. Rev., 114, 1-4, doi:10.1007/s11214-004-1406-4.

N. Achilleos and P. Guio, Atmospheric Physics Laboratory, University College London, Gower Street, London WC1E 6BT, UK. (nick@apl.ucl. ac.uk)

C. S. Arridge and A. J. Coates, Mullard Space Science Laboratory, Holmbury St. Mary, Dorking RH5 6NT, UK.

N. Sergis, Office for Space Research and Technology, Academy of Athens, Soranou Efesiou 4, GR-11527 Athens, Greece.

M. F. Thomsen and R. J. Wilson, Space Science and Applications, Los Alamos National Laboratory, PO Box 1663, MS D466, Los Alamos, NM 87545, USA. 